\title{
miR-21 facilitates the diagnostic value of miR-138 for papillary thyroid cancer in formalin-fixed paraffin-embedded tissues
}

\author{
Li Wang ${ }^{1 \#}$, Yuanyuan Duan ${ }^{1,2 \#}$, Wen Peng ${ }^{3}$, Caixing Qu ${ }^{4}$, Jiang Lin ${ }^{5}$, Zhaoqun Deng ${ }^{5}$, Chao You ${ }^{6,7}$, \\ Chenguang $\mathrm{Wu}^{1 \#}$
}

${ }^{1}$ Endocrinology Department, Affiliated People's Hospital, Jiangsu University, Zhenjiang 212002, China; ${ }^{2}$ Institute of Dermatology, Chinese Academy of Medical Sciences and Peking Union Medical College, Nanjing 210042, China; ${ }^{3}$ Department of Public Health, School of Medicine, Qinghai University, Xining 810001, China; ${ }^{4}$ Surgical Department, ${ }^{5}$ Central Lab, Affiliated People's Hospital, Jiangsu University, Zhenjiang 212002 , China; ${ }^{6}$ Department of Radiology, Fudan University Cancer Center, ${ }^{7}$ Department of Oncology, Shanghai Medical College, Fudan University, Shanghai 200032, China

Contributions: (I) Conception and design: L Wang, Y Duan, W Peng; (II) Administrative support: J Lin, Z Deng, C Wu; (III) Provision of study materials or patients: L Wang, Y Duan, W Peng, C Qu; (IV) Collection and assembly of data: Y Duan, W Peng; (V) Data analysis and interpretation: L Wang, Y Duan, W Peng; (VI) Manuscript writing: All authors; (VII) Final approval of manuscript: All authors.

"These authors contributed equally to this work.

Correspondence to: Chenguang Wu. Endocrinology Department, Affiliated People's Hospital, Jiangsu University, Zhenjiang 212001, China. Email: wcgzj1128@aliyun.com.

Background: miR-138 was thought to be anti-tumor miRNA for its ability of suppressing tumor growth. The objective of this study was to evaluate the potential predictive value of miR-138 and its combination with miR-21 in formalin-fixed paraffin-embedded (FFPE) tissues of papillary thyroid cancers (PTCs).

Methods: miR-138/miR-21 expression was tested in 101 PTC and 51 benign thyroid nodule (control) patients using quantitative real-time PCR. Clinical and pathological characteristics of enrolled subjects were obtained from medical records.

Results: miR-138 was significantly down-regulated in PTC compared with controls (median 0.0122 vs. 0.0943), and miR-138 exhibited capability of discriminating PTC from controls, with a diagnostic accuracy of $71.1 \%$ (sensitivity $84.3 \%$, specificity $49.5 \%$ ). A combination of miR-138 and miR-21 increased the diagnostic accuracy to $79.1 \%$ (sensitivity $73.3 \%$, specificity $76.5 \%$ ). Multivariate logistic regression confirmed the association between miR-138/miR-21 expression and PTC diagnosis. Moreover, miR-138 expression was further down-regulated in PTC with capsule invasion compared with PTC tissues without capsule invasion (median 0.0043 vs. $0.0373, \mathrm{P}=0.003$ ).

Conclusions: MiR-138 expression was not only associated with onset of PTC, but also the aggressiveness of PTC. A combination of miR-138 and miR-21 enhanced the diagnostic value of individual biomarkers.

Keywords: Biomarkers; papillary thyroid cancer (PTC); formalin-fixed paraffin-embedded tissue; miR-138; miR-21

Submitted Sep 25, 2018. Accepted for publication Jul 30, 2019.

doi: $10.21037 /$ tcr.2019.08.14

View this article at: http://dx.doi.org/10.21037/tcr.2019.08.14

\section{Introduction}

Thyroid cancer is the most common endocrinology cancer. In the United States, the incidence of new thyroid cancer cases was 13.9 per 100,000 men and women per year, based on the data 2009-2013, accounting for 3.8\% of new cancer cases (1). Among all the thyroid cancers, papillary thyroid cancer (PTC) is most common (1). In the primary test for thyroid cancer-fine needle aspiration biopsy (FNAB)-up to one third of the biopsy results were indeterminate, without clear indication for diagnosis (2). In this case, biomarkers to differentiate malignant and benign lesions are of our interest. 
MicroRNAs are small non-coding RNAs typically with 18-21 nucleotides $(3,4)$. MicroRNAs work in posttranscriptional gene silencing, thus triggering either translational repression or RNA degradation (5). In this way, miRNAs play important roles in many regulatory pathways including tumor growth (6) and invasion (7).

Normally fresh and frozen tissues are considered as proper samples for RNA research. However, given the time and money cost of prospectively fresh/frozen tissues collecting, formalin-fixed paraffin-embedded (FFPE) tissues were considered as potential alternative resource for biomedical research (8). Because of the shorter length of miRNAs, they seemed to be more stable and can be detected in FFPE tissues, when RNA appeared to degrade in the same samples (9). Meanwhile, miRNA expression in FFPE tissues was highly correlated with miRNA expression in matched fresh/frozen tissues (8). Therefore, given the comparative easy availability and low cost of obtaining FFPE tissues, they might be an ideal source for miRNA analysis.

miR-138 and miR-21 play vital roles in tumor biology. miR-138 was shown to suppress tumor growth (10-12), reverse drug resistance $(13,14)$ in cancers, and inhibit differentiation in stem cells $(15,16)$. In addition, miR-138 was also associated with epithelial-mesenchymal transition (16-18), which plays important role in cancer development and progression. On the contrary, miR-21 was suggested to promote cancer $(19,20)$. Recently, You et al. (21) have reported significant differences in expression levels of miR-21 and miR-138 when comparing colorectal cancer and normal adjacent tissues. Results showed that the expression level of miR-21 in colon cancer tissues was significantly higher comparing to adjacent healthy tissues, while miR-138 was lower in colon cancer tissues. And they indicated that miR-21 and miR-138 may be involved in the regulation of colon cancer cell proliferation. A study on the mechanism of prostate cancer metastasis showed that $\alpha$-solanine reversed EMT by down-regulating the expression of miR-21 and up-regulating the expression of miR-138, which may be involved in the anti-metastatic mechanisms of $\alpha$-solanine in human prostate cancer cell (22).

In thyroid cancers, miR-2 1 has been proved to be an oncogenic factor $(23,24)$, while miR-138 was much less studied. Several studies have reported overexpression of miR-21 in PTCs, as compared with levels in non-cancer thyroid tissues (23-25), and miR-21 was associated with the risk of recurrence of PTCs (26). MiR-21 was also reported to be significantly overexpressed in medullary thyroid cancer (MTC) by comparison with adjacent normal thyroid tissues (27). In addition, Chu et al. (28) indicated that increased expression of miR-21 was related to the worse prognosis with persistent and metastatic disease in MTC patients. More recently, Samsonov et al. (29) have identified that miR-21 was overexpressed in the plasma exosomes of patients with follicular thyroid cancer (FTC), than those in PTC patients, while miR-181a was higher expressed in PTC patients than in patients with FTC. And comparative assessment of miR-21-5p/miR-181a amplification ratios contributed to distinguish patients with FTC from patients with PTC with $100 \%$ sensitivity and relatively high specificity. In several studies, miR-138 was shown to be down-regulated in thyroid cancers (30-32). Of note, significantly reduced expression of miR-138 was observed in Hürthle cell thyroid cancer (HCTC), as well as patients with distant metastases, compared to normal thyroid tissues (33). While in very limited study, miR-138 was shown to be up-regulated in malignant thyroid cancers (34). To our knowledge, no reasonable explanation was given for that inconsistency. Furthermore, a racial disparity in miR138 expression was demonstrated by Suresh et al. (30). They found that miR-138 distributed differently in Caucasian Americans and African Americans between malignant and benign thyroid lesions. This study gave us a hint, that the possibility of different miR-138 distribution pattern might exist in Chinese population. Up to now, most of the studies on miR-138 were carried out in vitro studies, e.g., cell lines $(32)$, or in other populations $(30-32,34)$ but not in Chinese population.

On the other hand, since miR-138 and miR-21 play countered roles in cancer development and progression, and both are involved in common cancer regulatory net, for example, related to epithelial-mesenchymal transition $(18,35-37)$, we extrapolate that the combination of both miRNAs might work in different ways compared with individual miRNAs.

Therefore, the objectives of the present study were (I) to investigate the diagnostic role of miR-138 in Chinese PTC patients; (II) to explore the possible interaction between miR-138 and miR-21 in PTC patients.

\section{Methods}

\section{Patients and samples}

FFPE tissue samples from 101 PTC and 51 thyroid benign nodules (control) patients. FFPE samples were 
A

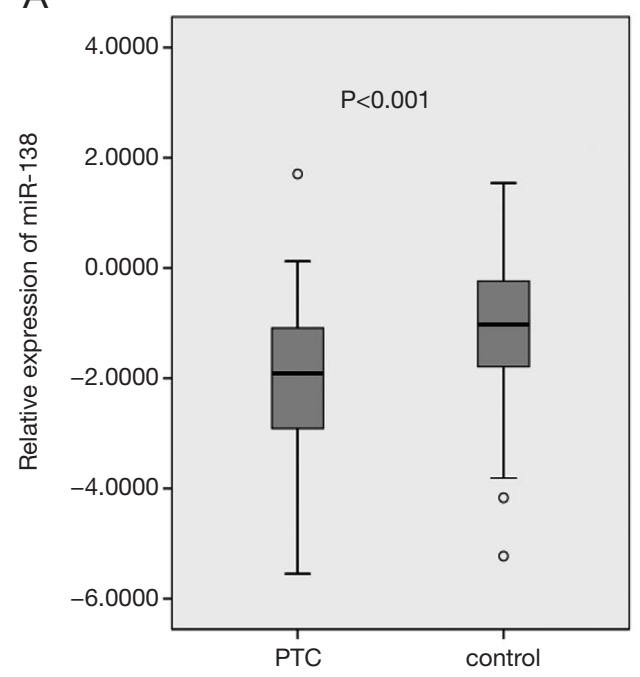

B

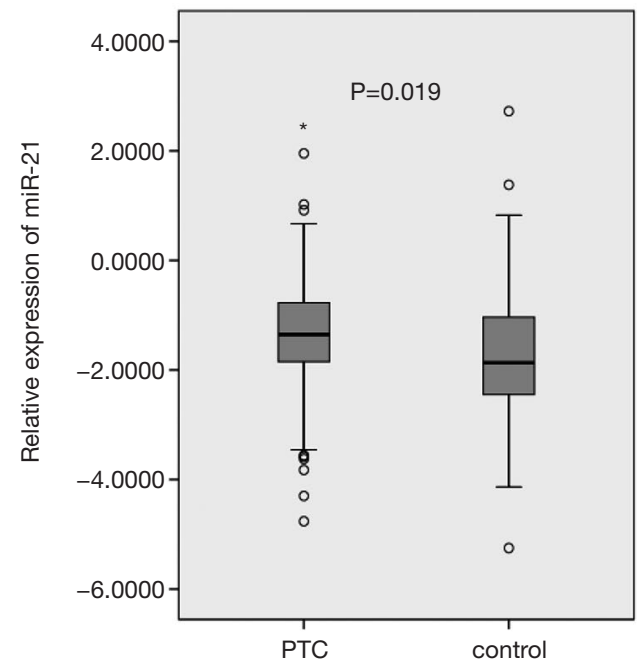

Figure 1 Relative miR-138 (A) and miR-21 (B) expression in 101 papillary thyroid cancers and 51 controls. miRNA expressions were presented after $\log 10$ transformation. Thick line in the box represented the median; upper- and lower-sides of box represented the $75 \%$ and $25 \%$ percentile; circles represented mild outliers; stars represented extreme outliers, error bars represented the maximum and minimum of "normal" distribution.

retrospectively collected from Affiliated People's Hospital of Jiangsu University. All samples were obtained based on the guidelines of hospital's protocol including patient consent and specimen collection. This study was approved by the Institutional Review Boards of Affiliated People's Hospital of Jiangsu University (No. 20150089). The diagnosis of thyroid cancers was based on pathological and clinical features of cancers.

\section{$R N A$ extraction and reverse transcription}

Total RNA was extracted in all the samples with the RecoverALL $^{\mathrm{TM}}$ Total Nucleic Acid Isolation Kit (Ambion, AM1975). Reverse transcription was made with miScript Reverse Transcription Kit (Qiagen, 218061). All the procedures were performed according to the instructions of manufacturers.

\section{Quantitative real-time PCR}

Quantitative real-time PCR was performed with miScript SYBR green PCR Kit (Qiagen, 218073), according to the protocol provided by the manufacturer. The primer in the Kit was used as universal forward primer. The sequences of reverse primers were: miR-138, 5'-AGCTGGTGTTGTGAATCAGGCCG-3'; miR-21,
5'-GAGCTTATCAGACTGATGTTGA-3'. All the miRNA expression was normalized by U6 expression. The condition of PCR was described in details before (38).

\section{Statistical methods}

All the statistical analyses were performed with SPSS 20.0. $\mathrm{miR}-138 / \mathrm{miR}-21$ expression was presented as median of copies normalized to U6 expression. Receiver operator characteristic (ROC) curves were drawn to show the diagnostic value of miRNA(s) for PTC. Univariate and multivariate logistic regressions were performed to analyze the association between miRNA expression and PTC. MiRNA expression in cancer patients by clinical and pathological characteristics was presented as quartile, and Mann-Whitney $\mathrm{U}$ test were applied to compare miRNA expression between groups.

\section{Results}

\section{miR-138/miR-21 expression in thyroid cancers and controls}

miR-138 exhibited significantly lower, and miR-21 showed significantly higher expression in PTC patients compared with controls (both $\mathrm{P}<0.05$ ) (Figure $1 A, B$; Table 1). The 
Table 1 Expression of miR-138/miR-21 and their diagnostic value for papillary thyroid cancer

\begin{tabular}{lcccccccc}
\hline miRNA & Median (cancer) & Median (control) & P & AUC & 95\% Cl & Cutoffs & Sensitivity (\%) & Specificity (\%) \\
\hline miR-138 & 0.0122 & 0.0943 & $<0.001$ & 0.711 & $0.624-0.798$ & 0.0099 & 84.3 & 49.5 \\
miR-21 & 0.0442 & 0.0136 & 0.019 & 0.617 & $0.520-0.713$ & 0.0138 & 76.2 & 51.0 \\
miR-21/miR-138 & 4.6447 & 0.1544 & $<0.001$ & 0.791 & $0.713-0.868$ & 1.0124 & 73.3 & 76.5 \\
\hline
\end{tabular}

The AUC of miR-138 was for controls. AUC, areas under the curve; $\mathrm{Cl}$, confidence interval
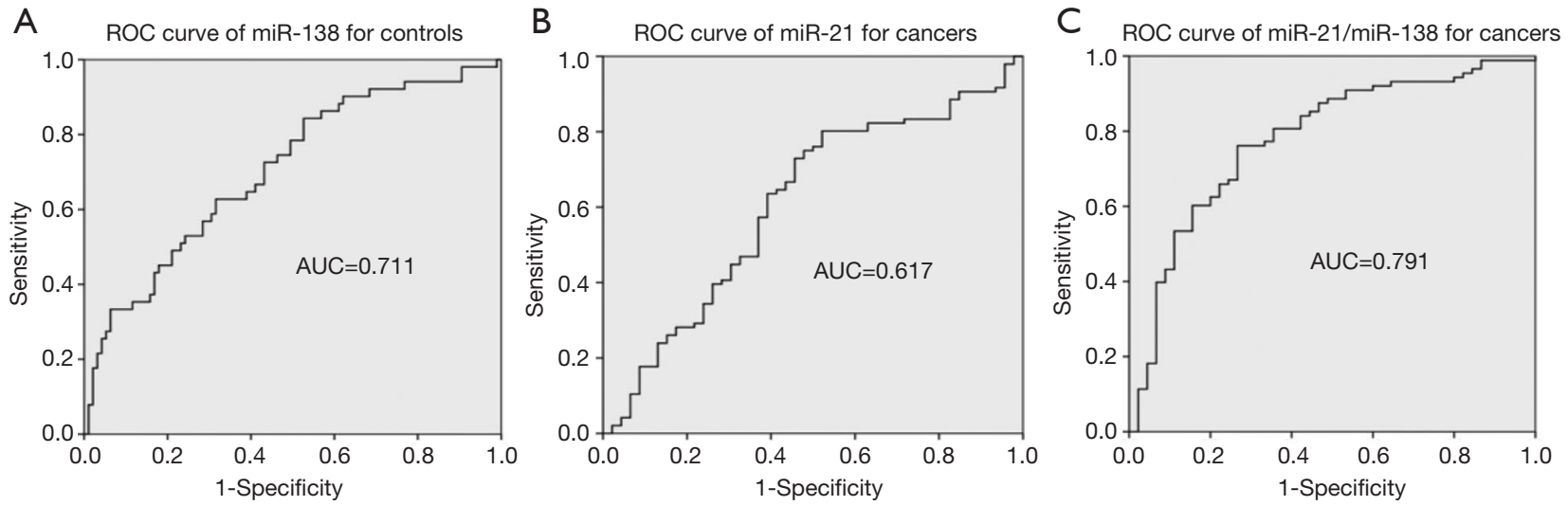

Figure 2 ROC curve of miR-138, miR-21 and the ratio of miR-21 and miR-138 expression. The area under the curve (AUC) indicated the accuracy for discriminating papillary thyroid cancer and controls in sensitivity and specificity. The combination of miR-138 and miR-2 1 produced a larger AUC than the individual miRNAs.

median of miR-138 expression in PTC patients was almost eight folds lower than that in controls (0.0122 vs. 0.0943), while the median of miR-21 expression in PTC patients was 3.3 times higher than that in controls (0.0442 vs. 0.0136)

(Table 1).

\section{Diagnostic value of miRNA-138/miR-21 for PTC}

ROC curve showed the capability of miRNA-138/miR-21 to discriminate PTC and controls. As indicated in Table 1 and Figure 2, both miR-138 and miR-21 had a significant discriminating capability between PTCs and controls (71.1\% and $61.7 \%$, respectively, both $\mathrm{P}<0.05)$. As shown in Table 1, with the optimized cut-offs, both miR-138 and miR-21 produced a relatively high sensitivity $(84.3 \%$ and $76.2 \%$, respectively), but only moderate specificity ( $49.5 \%$ and $51.0 \%$, respectively). However, a combination of both produced a higher diagnostic accuracy $(79.1 \%)$, as well as an increased specificity (76.5\%) and slightly decreased sensitivity $(73.3 \%)$ (Table 1$)$. In addition, multivariate logistic regression showed that miR-138 had a protective role $(\mathrm{OR}=0.456,95 \% \mathrm{CI}: 0.326-0.639)$ and $\mathrm{miR}-21 \mathrm{had}$ a predictive value $(\mathrm{OR}=1.874,95 \% \mathrm{CI}: 1.272-2.761)$ for PTC, despite that the univariate regression result for miR-21 was not significant $(\mathrm{P}=0.087)$ (Table 2).

\section{miR-138/miR-21 expression by clinical and pathological characteristics in PTC patients}

To further investigate whether miRNA expression was associated with aggressive features of PTC, we compared the miR-138 and miR-21 expression in cancer patients by clinical and pathological features. As shown in Table 3, miR-138 expression was further down-regulated in PTC with aggressive features, including advanced tumor stage (stage III, IV), capsule invasion, lymph node metastasis and extrathyroidal extension, only with statistical significance in PTC with capsule invasion $(\mathrm{P}=0.003)$. miR-21 expression did not differ by clinical and pathological characteristics.

\section{Discussion}

In the present study, we found that miR-138 expression in PTC was significantly down-regulated, while miR-21 was 
Table 2 Univariate and multivariate logistic regression of miR-138/miR-21 for papillary thyroid cancer

\begin{tabular}{|c|c|c|c|c|c|c|}
\hline Univariate/multivariate & $\beta$ & S.E. & Wald & $\mathrm{P}$ & OR & $95 \% \mathrm{Cl}$ \\
\hline miR-138 & -0.590 & 0.151 & 15.249 & $<0.001$ & 0.554 & $0.412-0.745$ \\
\hline miR-21 & 0.246 & 0.143 & 2.936 & 0.087 & 1.279 & $0.965-1.694$ \\
\hline \multicolumn{7}{|l|}{ Multivariate } \\
\hline miR-21 & 0.628 & 0.198 & 10.098 & 0.001 & 1.874 & $1.272-2.761$ \\
\hline
\end{tabular}

miRNA expression was log10 transformed in the logistic regression model. $\mathrm{Cl}$, confidence interval.

significantly up-regulated compared with benign thyroid nodules (both $\mathrm{P}<0.05$ ) (Table 1, Figure 1). Both miR-138 and miR-21 produced a significant diagnostic accuracy ( $71.1 \%$ vs. $61.7 \%$, respectively), and a combination of them produced a higher diagnostic accuracy $(79.1 \%)$ (Table 1, Figure 2). The logistic regression of miRNA expression for PTC confirmed the diagnostic value of the combination of both miRNAs, showing as the protective role of miR138 against $(\mathrm{OR}=0.456,95 \% \mathrm{CI}: 0.326-0.639, \mathrm{P}<0.001)$ and predictive value of miR-21 for PTC (OR $=1.874,95 \%$ CI: 1.272-2.761, $\mathrm{P}=0.001$ ) (Table 2). Further comparison of miRNA expression in PTC patients by clinical and pathological characteristics showed that miR-138 expression was significantly lower in cancers with capsule invasion (Table 3), suggesting that miR-138 was associated with aggressiveness of PTC.

First we analyzed the miR-138 and miR-21 expression in PTC and benign thyroid nodule tissues. The up-regulation of miR-21 expression in PTC in our data was consistent with previous studies $(23,25)$. Regarding the much less studied miR-138, in most cases, miR-138 was shown to be down-regulated in PTC patients (30-32), though limited study showed a up-regulation of miR-138 in thyroid cancers of follicular origin (34), and a racial disparity was also reported by Suresh et al. (30). Suresh's data agreed on the general down-regulation of miR-138 in PTC $(\mathrm{P}=0.0001)$, but to a larger magnitude in African Americans than in Caucasian Americans. Though with limitation of small sample size and lack of statistical significance, this study showed that ethnicity might be another confounding factor for miR-138 expression. In the present study, we found that miR-138 expression in Chinese PTC patients was eight times lower than that in benign thyroid nodules $(\mathrm{P}<0.001$, Table 1), which was consistent with Cong's study in another Chinese population (39). Our data supported the general opinion of down-regulated miR-138 in PTCs, and provided new evidences in Chinese population, corresponding to the racial disparity in miR-138 distribution. The diagnostic accuracy of miR-138 for PTC differed in studies, ranging from some $70 \%$ to $90 \%(34,39)$. In our data, the diagnostic accuracy was $71.1 \%$. It is worth noting that, for the first time, to our knowledge, we found that a combination of miR-138 and miR-21 could increase the diagnostic accuracy (in our case from $71.1 \%$ to $79.1 \%$ ); meanwhile, sensitivity and specificity were balanced to a more appropriate status for a diagnostic tool (sensitivity $73.3 \%$, specificity $76.5 \%$ ) (Table 1). Compared with univariate logistic regression, multivariate logistic regression revealed an intensified protective role of miR-138 (multivariate $v s$. univariate OR $=0.456 v s .0 .554)$ against and a stronger predictive role of miR-21 (multivariate $v s$. univariate $\mathrm{OR}=1.874 v s .1 .279$ ) for PTC (Table 2). Interestingly, the predictive role of miR-21 has turned to be statistically significant after controlling for miR138 (P value turned from 0.087 to 0.001 ) (Table 2). These results also supported the increased diagnostic accuracy of the combination. Furthermore, plasma exosomal miR-21 expression also helped to differentiate follicular from PTCs, demonstrated as the significantly higher miR-21 expression in FTCs (29).

The increased diagnostic accuracy of combined biomarkers (Table 1) and stronger association between combined biomarkers and PTC (Table 2) suggested a possible practical diagnostic tool in clinical setting. Since the high correlation of miRNA expression between FFPE samples and fresh/frozen samples, this diagnostic tool would help to differentiate PTC from benign nodules, particularly for those patients who had indeterminate FNAB results, thus decreasing unnecessary thyroid operations (40).

Furthermore, the down-regulated miR-138 expression in cancers with capsule invasion (Table 3) suggested that miR- 
Table 3 miR-138/miR-21 expression by clinical and pathological characteristics in papillary thyroid cancer

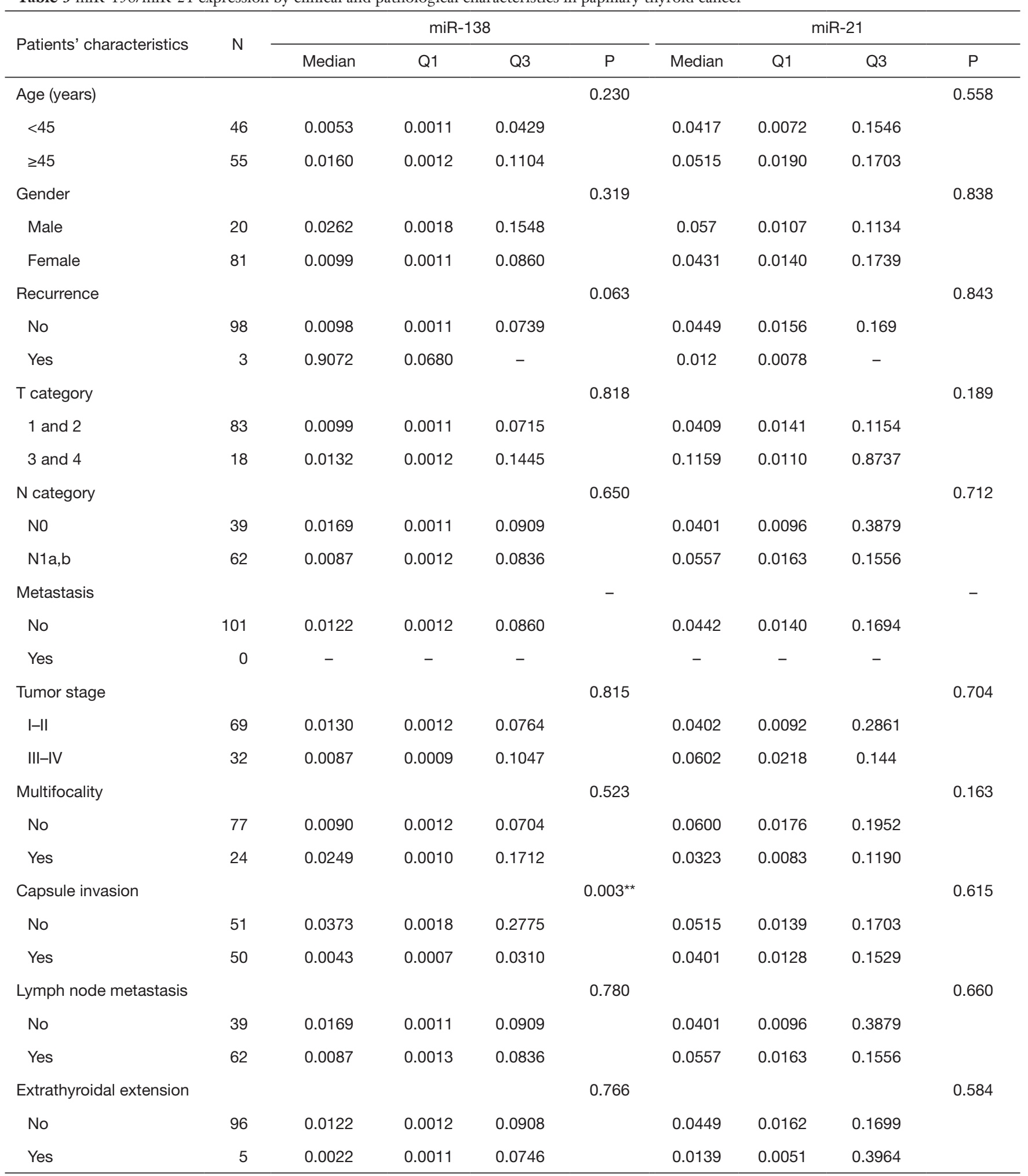

**, $\mathrm{P}<0.01$. 
138 dysregulation was not only associated with the onset of PTC, but also the aggressiveness of PTC. Similar results were also observed in Americans (31) and another Chinese population (39). In Yip's study, miR-138 expression in aggressive PTC was only half of nonaggressive PTC, but no statistical significance was seen $(\mathrm{P}=0.189)$ (31). In Cong's study, miR-138-1 expression was significantly lower in PTC with extrathyroidal extension $(\mathrm{P}=0.005)$ and in PTC patients with advanced tumor stage (III/IV stage, $\mathrm{P}=0.045$ ) (39). In our data, we did not find significant difference in miR-138 expression in PTC patients stratified by Tumor stage, lymph node metastasis or extrathyroidal extension, which are common indicators for aggressiveness of PTC. However, miR-138 expression in PTC samples with these "aggressive" features were lower than those without these features, in spite of lack of statistical difference. The lack of significance might be caused by the characteristics of enrolled patients and limited sample size. In our study, for most cases, PTC was diagnosed in routine test and exams, and patients enrolled were generally mild cases. The relative small number of "aggressive" tumors may hide the difference of miR-138 expression. However, the significant difference in miR-138 expression between patients with/without capsule invasion has supported the miR-138 role in the aggressiveness of cancers.

On the other hand, in vitro study, miR-138 expression in anaplastic thyroid cancer, a more aggressive type of thyroid cancer, was also down-regulated compared with PTC, a relative mild type of thyroid cancer (32). This finding supported the aggressive feature of miR-138 down-regulated thyroid cancers, though from comparison between pathological types.

\section{Conclusions}

In conclusion, we revealed that miR-138 down-regulation was not only associated with onset of PTC, but also the aggressiveness of PTC. And for the first time, we showed that a combination of miR-138 and miR-21 could increase the diagnostic accuracy for PTC. A combination of miR138 and miR-21 could be used as a diagnostic tool to differentiate PTC from benign lesions. Further studies will be carried out to disclose the mechanism of miRNA-138 to regulate tumor genesis and progression, and the interaction with miR-21.

\section{Acknowledgments}

Funding: The study was sponsored by Natural Science foundation of Jiangsu Province, China (BK20150476), a grant to $W$ Peng for innovative projects from Jiangsu Province and Zhenjiang scientific and technological supportive plan (social development) (SH2017028, FZ2014031).

\section{Footnote}

Conflicts of Interest: All authors have completed the ICMJE uniform disclosure form (available at http://dx.doi. org/10.21037/tcr.2019.08.14). The authors have no conflicts of interest to declare.

Ethical Statement: The authors are accountable for all aspects of the work in ensuring that questions related to the accuracy or integrity of any part of the work are appropriately investigated and resolved. This study was conducted in accordance with the Declaration of Helsinki (as revised in 2013). This retrospective study was approved by the ethics committee of the Affiliated People's Hospital of Jiangsu University (No. 20150089). All samples were obtained based on the guidelines of hospital's protocol including patient consent and specimen collection.

Open Access Statement: This is an Open Access article distributed in accordance with the Creative Commons Attribution-NonCommercial-NoDerivs 4.0 International License (CC BY-NC-ND 4.0), which permits the noncommercial replication and distribution of the article with the strict proviso that no changes or edits are made and the original work is properly cited (including links to both the formal publication through the relevant DOI and the license). See: https://creativecommons.org/licenses/by-nc-nd/4.0/.

\section{References}

1. Cancer Stats Facts: Thyroid Cancer Available online: https://seer.cancer.gov/statfacts/html/thyro.html, accessed on July 14th, 2017.

2. Dhyani M, Faquin W, Lubitz CC, et al. How to interpret thyroid fine-needle aspiration biopsy reports: a guide for the busy radiologist in the era of the 
Bethesda Classification System. AJR Am J Roentgenol 2013;201:1335-9.

3. Hutvágner G, Zamore PD. A microRNA in a multiple-turnover RNAi enzyme complex. Science 2002;297:2056-60.

4. Lund E, Guttinger S, Calado A, et al. Nuclear export of microRNA precursors. Science 2004;303:95-8.

5. Seitz H, Youngson N, Lin SP, et al. Imprinted microRNA genes transcribed antisense to a reciprocally imprinted retrotransposon-like gene. Nat Genet 2003;34:261-2.

6. Lee DY, Deng Z, Wang CH, et al. MicroRNA-378 promotes cell survival, tumor growth, and angiogenesis by targeting SuFu and Fus-1 expression. Proc Natl Acad Sci U S A 2007;104:20350-5.

7. Du WW, Fang L, Li M, et al. MicroRNA miR-24 enhances tumor invasion and metastasis by targeting PTPN9 and PTPRF to promote EGF signaling. J Cell Sci 2013;126:1440-53.

8. Goswami RS, Waldron L, Machado J, et al. Optimization and analysis of a quantitative real-time PCR-based technique to determine microRNA expression in formalinfixed paraffin-embedded samples. BMC Biotechnol 2010;10:47.

9. Li X, Lu Y, Chen Y, et al. MicroRNA profile of paclitaxelresistant serous ovarian carcinoma based on formalin-fixed paraffin-embedded samples. BMC Cancer 2013;13:216.

10. Erdmann K, Kaulke K, Rieger C, et al. MiR-26a and miR138 block the G1/S transition by targeting the cell cycle regulating network in prostate cancer cells. J Cancer Res Clin Oncol 2016;142:2249-61.

11. Golubovskaya VM, Sumbler B, Ho B, et al. MiR-138 and MiR-135 directly target focal adhesion kinase, inhibit cell invasion, and increase sensitivity to chemotherapy in cancer cells. Anticancer Agents Med Chem 2014;14:18-28.

12. Ye XW, Yu H, Jin YK, et al. miR-138 inhibits proliferation by targeting 3-phosphoinositide-dependent protein kinase-1 in non-small cell lung cancer cells. Clin Respir J 2015;9:27-33.

13. Zhao X, Yang L, Hu J, et al. miR-138 might reverse multidrug resistance of leukemia cells. Leuk Res 2010;34:1078-82.

14. Gao $Y$, Fan $\mathrm{X}, \mathrm{Li} W$, et al. miR-138-5p reverses gefitinib resistance in non-small cell lung cancer cells via negatively regulating $\mathrm{G}$ protein-coupled receptor 124 . Biochem Biophys Res Commun 2014;446:179-86.

15. Yang Z, Bian C, Zhou H, et al. MicroRNA hsa-miR-138 inhibits adipogenic differentiation of human adipose tissue-derived mesenchymal stem cells through adenovirus
EID-1. Stem Cells Dev 2011;20:259-67.

16. Eskildsen T, Taipaleenmaki H, Stenvang J, et al. MicroRNA-138 regulates osteogenic differentiation of human stromalmesenchymal. stem cells in vivo. Proc Natl Acad Sci U S A 2011;108:6139-44.

17. Cai Q, Wang Z, Wang S, et al. Long non-coding RNA LINC00152 promotes gallbladder cancer metastasis and epithelial-mesenchymal transition by regulating HIF1alpha via miR-138. Open Biol 2017. doi: 10.1098/ rsob.160247.

18. Li J, Wang Q, Wen R, et al. MiR-138 inhibits cell proliferation and reverses epithelial-mesenchymal transition in non-small cell lung cancer cells by targeting GIT1 and SEMA4C. J Cell Mol Med 2015;19:2793-805.

19. Peralta-Zaragoza O, Deas J, Meneses-Acosta A, et al. Relevance of miR-21 in regulation of tumor suppressor gene PTEN in human cervical cancer cells. BMC Cancer 2016;16:215.

20. Tian L, Shan W, Zhang Y, et al. Up-regulation of miR-21 expression predicate advanced clinicopathological features and poor prognosis in patients with non-small cell lung cancer. Pathol Oncol Res 2016;22:161-7.

21. You C, Jin L, Xu Q, et al. Expression of miR-21 and miR138 in colon cancer and its effect on cell proliferation and prognosis. Oncol Lett 2019;17:2271-7.

22. Shen KH, Liao AC, Hung JH, et al. $\alpha$-Solanine inhibits invasion of human prostate cancer cell by suppressing epithelial-mesenchymal transition and MMPs expression. Molecules 2014;19:11896-914.

23. Sondermann A, Andreghetto FM, Moulatlet AC,et al. MiR-9 and miR-21 as prognostic biomarkers for recurrence in papillary thyroid cancer. Clin Exp Metastasis 2015;32:521-30.

24. Huang YH, Lin YH, Chi HC, et al. Thyroid hormone regulation of miR-21 enhances migration and invasion of hepatoma. Cancer Res 2013;73:2505-17.

25. Yoruker EE, Terzioglu D, Teksoz S, et al. MicroRNA expression profiles in papillary thyroid carcinoma, benign thyroid nodules and healthy controls. J Cancer 2016;7:803-9.

26. Rosignolo F, Memeo L, Monzani F, et al. MicroRNAbased molecular classification of papillary thyroid carcinoma. Int J Oncol 2017;50:1767-77.

27. Mian C, Pennelli G, Fassan M, et al. MicroRNA profiles in familial and sporadic medullary thyroid carcinoma: preliminary relationships with RET status and outcome. Thyroid 2012;22:890-6.

28. Chu YH, Lloyd RV. Medullary Thyroid Carcinoma: 
Recent Advances Including MicroRNA Expression. Endocr Pathol 2016;27:312-24.

29. Samsonov R, Burdakov V, Shtam T, et al. Plasma exosomal miR-21 and miR-181a differentiates follicular from papillary thyroid cancer. Tumour Biol 2016;37:12011-21.

30. Suresh R, Sethi S, Ali S, et al. Differential expression of MicroRNAs in papillary thyroid carcinoma and their role in racial disparity. J Cancer Sci Ther 2015;7:145-54.

31. Yip L, Kelly L, Shuai Y, et al. MicroRNA signature distinguishes the degree of aggressiveness of papillary thyroid carcinoma. Ann Surg Oncol 2011;18:2035-41.

32. Mitomo S, Maesawa C, Ogasawara S, et al.

Downregulation of miR-138 is associated with overexpression of human telomerase reverse transcriptase protein in human anaplastic thyroid carcinoma cell lines. Cancer Sci 2008;99:280-6.

33. Petric R, Gazic B, Goricar K, et al. Expression of miRNA and Occurrence of Distant Metastases in Patients with Hürthle Cell Carcinoma. Int J Endocrinol 2016;2016:8945247.

34. Vriens MR, Weng J, Suh I, et al. MicroRNA expression profiling is a potential diagnostic tool for thyroid cancer. Cancer 2012;118:3426-32.

35. Han M, Liu M, Wang Y, et al. Re-expression of miR-

Cite this article as: Wang L, Duan Y, Peng W, Qu C, Lin J, Deng Z, You C, Wu C. miR-21 facilitates the diagnostic value of miR-138 for papillary thyroid cancer in formalin-fixed paraffin-embedded tissues. Transl Cancer Res 2019;8(5):1718-1726. doi: $10.21037 /$ tcr.2019.08.14
21 contributes to migration and invasion by inducing epithelial-mesenchymal transition consistent with cancer stem cell characteristics in MCF-7 cells. Mol Cell Biochem 2012;363:427-36.

36. Liu CH, Huang Q, Jin ZY, et al. miR-21 and KLF4 jointly augment epithelial mesenchymal transition via the Akt/ ERK1/2 pathway. Int J Oncol 2017;50:1109-15.

37. Jin Z, Guan L, Song Y, et al. MicroRNA-138 regulates chemoresistance in human non-small cell lung cancer via epithelial mesenchymal transition. Eur Rev Med Pharmacol Sci 2016;20:1080-6.

38. Deng Z, Du WW, Fang L, et al. The intermediate filament vimentin mediates microRNA miR-378 function in cellular self-renewal by regulating the expression of the Sox2 transcription factor. J Biol Chem 2013;288:319-31.

39. Cong D, He M, Chen S, et al. Expression profiles of pivotal microRNAs and targets in thyroid papillary carcinoma: an analysis of The Cancer Genome Atlas. Onco Targets Ther 2015;8:2271-7.

40. Kitano M, Rahbari R, Patterson EE, et al. Expression profiling of difficult-to-diagnose thyroid histologic subtypes shows distinct expression profiles and identify candidate diagnostic microRNAs. Ann Surg Oncol 2011;18:3443-52. 